\title{
Clinical characteristics and risk factors of non-Candida fungaemia
}

\author{
Masaki Yamamoto', Shunji Takakura", Gou Hotta', Yasufumi Matsumura', Aki Matsushima', Miki Nagao', \\ Yutaka Ito ${ }^{2}$ and Satoshi Ichiyama'
}

\begin{abstract}
Background: The incidence of fungaemia has been increasing worldwide. It is important to distinguish nonCandida fungaemia from candidaemia because of their different antifungal susceptibilities. The aims of this study were to investigate the clinical characteristics of non-Candida fungaemia and identify the clinical factors that differentiate it from candidaemia.

Methods: We investigated the clinical manifestations and mortality of non-Candida fungaemia in Kyoto University Hospital from 2004 to 2009.

Results: There were 110 episodes of fungaemia during the study period. There were 11 renal replacement therapy episodes of fungaemia due to non-Candida yeasts (10.0\%), including 6 episodes with Cryptococcus neoformans, 4 with Trichosporon asahii, and 1 with Kodamaea ohmeri, in addition to 99 episodes of candidaemia (90.0\%). The presence of collagen disease [odds ratio (OR) 9.00; 95\% confidence interval (CI) 1.58-51.4; $P=0.01$ ] or renal replacement therapy (OR 15.0; 95\% Cl 3.06-73.4; $P<0.01)$ was significantly more common in non-Candida fungaemia patients than in candidaemia patients. Prior colonisation by the species may be a predictor of nonCandida fungaemia. Non-Candida fungaemia had a higher mortality than candidaemia (54.5\% versus $21.2 \%$, $P=0.03)$.

Conclusions: Although Candida species frequently cause fungaemia, we should also be aware of non-Candida yeasts because of their high mortality, particularly among high-risk patients, such as those with collagen disease and those under renal replacement therapy. Prior colonisation by the causative organisms may be an important predictor of non-Candida fungaemia.
\end{abstract}

Keywords: Fungaemia, Non-Candida yeast, Risk factor, Mortality, Colonisation

\section{Background}

The incidence of hospital-acquired fungaemia caused by yeasts has increased dramatically during the past two decades [1]. This increased incidence has been associated with advances in clinical medicine, including organ transplantation, chemotherapy, antimicrobial agents, parenteral nutrition, and medical devices, all of which improve patient survival but increase the risk of infection [2]. Candida species are the leading cause of yeast fungaemia. However, as the fungaemia patient population has changed, several types of rare yeasts have become

\footnotetext{
* Correspondence: stakakr@kuhp.kyoto-u.ac.jp

'Department of Clinical Laboratory Medicine, Kyoto University Graduate School of Medicine, 54 Shogoin-Kawaharacho, Sakyo-ku, Kyoto 6068507 Japan

Full list of author information is available at the end of the article
}

recognised pathogens, particularly in nosocomial settings, and have increased in clinical importance [1]. These yeasts, which include Trichosporon species, Cryptococcus species, Rhodotorula species, Malassezia species, and Blastoschizomyces capitatus, have been associated with life-threatening infections in immunocompromised patients [3-6].

The mortality rate among patients with fungaemia is high, ranging from $50 \%$ to $80 \%$ [1,7-10]. The variable susceptibility profiles to antifungal agents are one of the major reasons for the poor prognosis of these yeast infections. Candida species are usually susceptible to standard antifungal agents. However, the treatment of non-Candida yeasts is challenging because of their rarity and the prevalence of in vitro resistance to standard
C Biomed Central

(c) 2013 Yamamoto et al.; licensee BioMed Central Ltd. This is an Open Access article distributed under the terms of the Creative Commons Attribution License (http://creativecommons.org/licenses/by/2.0), which permits unrestricted use, distribution, and reproduction in any medium, provided the original work is properly cited. 
antifungal agents [11]. For example, Cryptococcus species are resistant to echinocandins, and Trichosporon species are characterised by resistance to amphotericin and echinocandins. For these reasons, early distinction between non-Candida species and Candida species is important.

Although the importance of non-Candida yeasts is recognised, little is known about the epidemiology and risk factors associated with non-Candida fungaemia. To evaluate the clinical characteristics of non-Candida fungaemia and determine the risk factors associated with non-Candida fungaemia, we conducted a retrospective cohort study of yeast fungaemia detected in a tertiary-care university hospital over a 6-year period.

\section{Methods}

\section{Study population}

From 2004 to 2009, all patients with non-Candida fungaemia were identified through the records of the clinical microbiological laboratory at Kyoto University Hospital, a tertiary-care, 1182-bed university hospital in Japan. The medical records of all patients with yeast fungaemia were reviewed. Patients were included if they had evidence of sepsis with at least one positive blood culture containing yeasts. Only one episode per patient was included in this study. The Institutional Review Board of Kyoto University Hospital approved this study protocol.

\section{Clinical characteristics and risk factor analysis}

Demographic data, the potential risk factors for developing yeast fungaemia presenting within 30 days prior to the diagnosis of fungaemia, and outcomes were retrieved from the medical records. The following data were recorded: age, gender, hospital-acquired infection (HAI), days of hospitalisation prior to the onset of fungaemia, admission ward at the onset of fungaemia, co-morbidities (solid malignancy, diabetes mellitus, gastrointestinal/ hepatobiliary disease, chronic kidney disease, haematologic malignancy, cardiovascular disease, and collagen disease), underlying conditions [prior intensive care unit admission, neutropenia, colonisation by the causative yeast, polymicrobial bacteraemia, assisted ventilation, renal replacement therapy (haemodialysis and continuous haemodiafiltration), indwelling Foley catheter, central venous catheterisation, parenteral nutrition, enteral nutrition, corticosteroid therapy, chemotherapy, other immunosuppressant therapy, prior antibiotic therapy, prior antifungal therapy, prior surgical procedures (abdominal or other), the severity of illness, and 30-day mortality.

Species identification and antifungal susceptibility testing Blood cultures were performed using the BacT/ALERT automated culture system (bioMerieux, Marcy-l'Etoile,
France). Species identification was performed with standard laboratory procedures, including morphological identification and the API 20C AUX system (bioMerieux). The minimum inhibitory concentrations (MICs) of each antifungal drug were determined using the reference broth microdilution method and interpreted according to the M27-A2 guidelines of the Clinical and Laboratory Standards Institute [12].

\section{Definitions}

HAI was defined as an infection acquired at least 48 hours after hospitalisation that was not clinically apparent at the time of hospitalisation. Neutropenia was defined as an absolute neutrophil count of less than 500 cells $/ \mu \mathrm{L}$. Polymicrobial bacteraemia was defined as the isolation of other bacteria from the blood within 24 hours of the initial positive fungal culture. Fungal colonisation was defined as a positive culture of causative yeasts from any bodily site other than blood before the onset of fungaemia, with no clinical sign or symptom of infection at that site. Corticosteroid therapy was defined as administration of at least $20 \mathrm{mg}$ of a prednisone equivalent for at least 1 week (this value was adjusted according to age for paediatric patients). The severity of illness was estimated using the sequential organ failure assessment (SOFA) score on the day of fungaemia onset.

\section{Statistics}

Statistical analyses were performed using SPSS version 18.0 (SPSS Inc., Chicago, IL, USA). Fisher's exact or Pearson's chi-square test was used as appropriate to compare categorical variables. The Mann-Whitney $U$ test was used to test for the statistical significance of continuous variables. Multivariate logistic regression analysis was used for risk factor analysis for non-Candida fungaemia and outcome analysis. A forward selection method was used with the entry criterion of a $P$ value $<0.1$ for clinical factors associated with risk factors of non-Candida fungaemia, and factors with a $P$ value $<0.05$ were retained in the final model. A $P$ value of $<0.05$ was considered statistically significant.

\section{Results}

During the study period, 102 yeasts were isolated from 110 patients with fungaemia. No patient had evidence of human immunodeficiency virus infection. Eleven of 110 episodes (10.0\%) were non-Candida fungaemia. Of the 110 episodes of yeast fungaemia, 112 yeast isolates were identified; 2 of 110 episodes showed coinfection with 2 Candida isolates. Among these yeast isolates, 11 (9.8\%) were non-Candida yeasts, and 101 (90.2\%) were Candida species. Among the 11 non-Candida isolates, Cryptococcus neoformans (6 isolates, 5.4\%), Trichosporon asahii (4 isolates, 3.6\%), and Kodamaea ohmeri (1 isolate, 
0.9\%) were identified. Among the 101 Candida isolates, the most common was Candida albicans (46 isolates, 41.1\%), followed by Candida parapsilosis (22 isolates, 19.6\%), Candida glabrata (15 isolates, 13.4\%), Candida tropicalis (9 isolates, 8.0\%), and Candida guilliermondii (5 isolates, 4.5\%). Candida krusei, Candida lusitaniae, and Candida famata were isolated in 1 episode each. Another Candida isolate could not be identified to the species level.

All 6 C. neoformans isolates showed low MIC values for amphotericin (0.125-0.25 $\mathrm{mg} / \mathrm{L})$ and fluconazole (1-8 $\mathrm{mg} / \mathrm{L})$ and high MIC values for micafungin ( $\geq 32 \mathrm{mg} / \mathrm{L}$ ). In the 4 isolates of $T$. asahii, the MIC values for fluconazole ranged from $4 \mathrm{mg} / \mathrm{L}$ to $8 \mathrm{mg} / \mathrm{L}$, and the MIC values for micafungin were $\geq 16 \mathrm{mg} / \mathrm{L}$. K. ohmeri showed low MIC values for amphotericin $(0.25 \mathrm{mg} / \mathrm{L})$, fluconazole $(\leq 0.125 \mathrm{mg} / \mathrm{L})$, and micafungin $(0.25 \mathrm{mg} / \mathrm{L})$. Nine of the 112 isolates of Candida species were resistant to fluconazole (8.0\%).

The medical records were available for all 110 patients with fungaemia. Table 1 shows the clinical characteristics of patients with non-Candida fungaemia. Immunosuppressants were used in 7 cases $(63.6 \%)$. Five patients (45.5\%) were under renal replacement therapy. Colonisation by causative organisms was identified in 8 patients (72.7\%), and urine was the major site of colonisation. Initial treatment with antifungal agents at a high MIC was found in 3 of 4 episodes of $T$. asahii fungaemia. Two episodes were treated with micafungin, and 1 was treated with fluconazole. In cryptococcaemia, a lack of antifungal therapy was identified in 2 cases because these 2 patients died before starting antifungal therapy, and others were treated with antifungal agents at a low MIC. A treatment delay of over 2 days or lack of antifungal therapy was noted for 8 patients $(72.7 \%)$.

The demographic characteristics and clinical manifestations of the patients with fungaemia are shown in Table 2. The median age of non-Candida fungaemia patients was 66 years (interquartile range 60-71 years), and $45.5 \%$ were female. There was no significant difference in age $(P=0.80)$, gender $(P=0.57)$, frequency of HAI $(P=0.65)$, or days of hospitalisation prior to fungaemia onset $(P=0.62)$ between the non-Candida fungaemia and candidaemia groups. Non-Candida fungaemia had a higher mortality rate than candidaemia (54.5\% versus $21.2 \%, P=0.03$ ).

The risk factors in patients with yeast fungaemia are shown in Table 3. In the univariate analysis, factors significantly associated with non-Candida fungaemia were chronic kidney disease [odds ratio (OR) 5.40; 95\% confidence interval (CI) 1.48-19.7; $P=0.01]$, prior bacteraemia (OR 3.66; 95\% CI 1.00-13.4; $P=0.04$ ), renal replacement therapy (OR 8.33; 95\% CI 2.12-32.8; $P<0.01$ ), corticosteroid therapy (OR 3.84; 95\% CI 1.05-14.1; $P=$ 0.04), and higher SOFA score (OR 1.13; 95\% CI 1.03-1.26; $P=0.02)$. In the multivariate analysis, independent risk factors associated with non-Candida fungaemia included collagen disease (OR 9.00; 95\% CI 1.58-51.4; $P=0.01$ ) and renal replacement therapy (OR 15.0; 95\% CI 3.06-73.4; $P<0.01)$.

\section{Discussion}

Non-Candida yeasts, which are clinically less common than Candida species, have been associated with lifethreatening infections in immunocompromised individuals. Although the importance of these opportunistic yeasts is recognised, little is known about their epidemiology [11]. In this study, similar to previous studies, fungaemia due to non-Candida yeasts was less common than candidaemia, but it still accounted for a significant proportion of all fungaemia episodes (10.0\%). This incidence was similar to the rate of fluconazole-resistant Candida among candidaemia in our hospital (8.0\%). The incidence of non-Candida fungaemia was slightly higher than in previous reports, which have reported rates of $3-6 \%[2,3]$. One possible reason for this finding is that we included fungaemia caused by $C$. neoformans in this study because of its different susceptibility profile to antifungal agents compared to Candida species. Cryptococcus species are resistant to echinocandins [13]. However,

Table 1 Clinical characteristics of non-Candida fungaemia infection

\begin{tabular}{|c|c|c|c|}
\hline & C. neoformans $(n=6)$ & T. asahii $(n=4)$ & K. ohmeri $(n=1)$ \\
\hline Co-morbidities $^{a}$ & $6(100 \%)$ & $4(100 \%)$ & $1(100 \%)$ \\
\hline Immunosuppressant therapy & $4(66.7 \%)$ & $2(50.0 \%)$ & $1(100 \%)$ \\
\hline Renal replacement therapy & $2(33.3 \%)$ & $2(50.0 \%)$ & $1(100 \%)$ \\
\hline Colonisation by causative yeasts & $4(66.7 \%)$ & $4(100 \%)$ & $1(100 \%)$ \\
\hline SOFA score, median (range) & $9(1-18)$ & $8(1-17)$ & $20(-)$ \\
\hline In vitro-active antifungal agent & $4(66.7 \%)$ & $1(25.0 \%)$ & $1(100 \%)$ \\
\hline 30-day mortality & $3(50.0 \%)$ & $3(75.0 \%)$ & $0(0 \%)$ \\
\hline
\end{tabular}

SOFA: sequential organ failure assessment.

${ }^{a}$ Co-morbidities of $C$. neoformans infection include acute respiratory distress syndrome, hepatitis $C$ virus infection, post-living donor liver transplantation (LDLT), systemic lupus erythaematosus, and miliary tuberculosis. Co-morbidities of $T$. asahii infection include POEMS syndrome, liver cirrhosis, Bechet's disease, and acute myeloid leukaemia. Co-morbidities of K. ohmeri infection include alcoholic liver cirrhosis and post-LDLT. 
Table 2 Demographic characteristics and treatment outcomes of patients with fungaemia $(N=110)$

\begin{tabular}{|c|c|c|c|c|}
\hline \multirow[t]{2}{*}{ Characteristics } & \multicolumn{3}{|c|}{ Type of fungaemia, $N(\%)$} & \multirow[t]{2}{*}{$P$ value } \\
\hline & Non-Candida fungaemia & Candidaemia & Overall & \\
\hline & $11(10.0)$ & $99(90.0)$ & $110(100)$ & \\
\hline Age, years ${ }^{a}$ & $66(60-71)$ & $65(49-74)$ & $65(54-73)$ & 0.80 \\
\hline Female gender & $5(45.5)$ & $54(54.5)$ & $59(53.6)$ & 0.57 \\
\hline Hospital-acquired infection & $11(100)$ & $95(96.0)$ & $106(96.4)$ & 0.65 \\
\hline Days of prior hospitalisation ${ }^{\mathrm{a}, \mathrm{b}}$ & $47(11-66)$ & $30(12-60)$ & $30(12-60)$ & 0.62 \\
\hline Admission ward at fungaemia onset & & & & 0.71 \\
\hline Surgical ward & $3(27.3)$ & $40(40.4)$ & $43(39.1)$ & \\
\hline Medical ward & $6(54.5)$ & $41(41.4)$ & $47(42.7)$ & \\
\hline $\mathrm{ICU}$ & $2(18.2)$ & $14(14.1)$ & $16(14.5)$ & \\
\hline Co-morbidities & $11(100)$ & $92(92.9)$ & $103(93.6)$ & 0.47 \\
\hline Solid malignancy & $0(0)$ & $45(45.5)$ & $45(40.9)$ & $<0.01^{*}$ \\
\hline Diabetes mellitus & $5(45.5)$ & $25(25.3)$ & $30(27.3)$ & 0.14 \\
\hline Gastrointestinal/hepatobiliary disease & $6(54.5)$ & $60(60.6)$ & $66(60.0)$ & 0.47 \\
\hline Chronic kidney disease & $6(54.5)$ & $18(18.2)$ & $24(21.8)$ & $0.01^{*}$ \\
\hline Haematologic malignancy & $1(9.1)$ & $14(14.1)$ & 15 (13.6) & 0.54 \\
\hline Cardiovascular disease & $3(27.3)$ & $17(17.2)$ & $20(18.2)$ & 0.32 \\
\hline Collagen disease & $3(27.3)$ & $9(9.1)$ & $12(10.9)$ & $0.09^{*}$ \\
\hline \multicolumn{5}{|l|}{ Underlying conditions } \\
\hline Prior ICU admission & $5(45.5)$ & $31(31.3)$ & $36(32.7)$ & 0.27 \\
\hline Neutropaenia & $1(9.1)$ & $7(7.1)$ & $8(7.3)$ & 0.58 \\
\hline Prior bacteraemia & $7(63.6)$ & $32(32.3)$ & $39(35.5)$ & $0.04^{*}$ \\
\hline Colonisation by causative yeasts & $8(72.7)$ & $41(41.4)$ & $49(44.5)$ & $0.05^{*}$ \\
\hline Polymicrobial bacteraemia & $5(45.5)$ & $23(23.2)$ & $28(25.5)$ & 0.11 \\
\hline Assisted ventilation & $2(18.2)$ & $17(17.2)$ & $19(17.3)$ & 0.60 \\
\hline Renal replacement therapy & $5(45.5)$ & $9(9.1)$ & $14(12.7)$ & $<0.01^{*}$ \\
\hline Indwelling Foley catheter & $7(63.6)$ & $42(42.4)$ & $49(44.5)$ & 0.15 \\
\hline Central venous catheterisation & $8(72.7)$ & $82(82.8)$ & $90(8.18)$ & 0.32 \\
\hline Parenteral nutrition & $8(72.7)$ & $80(80.8)$ & $88(80.0)$ & 0.38 \\
\hline Enteral nutrition & $9(81.8)$ & $56(56.6)$ & $65(59.1)$ & $0.09^{*}$ \\
\hline Corticosteroid therapy & $7(63.6)$ & $31(31.3)$ & $38(34.5)$ & $0.04^{*}$ \\
\hline Chemotherapy & $0(0)$ & $27(27.3)$ & $27(24.5)$ & $0.04^{*}$ \\
\hline Other immunosuppressant therapy & $3(27.3)$ & $8(8.1)$ & $11(10.0)$ & $0.08^{*}$ \\
\hline Prior antibiotic therapy & $10(90.9)$ & 89 (89.9) & $99(90.0)$ & 0.70 \\
\hline Prior antifungal therapy & $4(36.4)$ & $18(18.2)$ & $22(20.0)$ & 0.15 \\
\hline Prior surgical procedures & $2(18.2)$ & $28(28.3)$ & $30(27.3)$ & 0.38 \\
\hline Abdominal surgery & $1(9.1)$ & $19(19.2)$ & $20(18.2)$ & 0.37 \\
\hline Other surgery & $1(9.1)$ & $10(10.1)$ & $11(10.0)$ & 0.70 \\
\hline SOFA score ${ }^{a}$ & $11(3-17)$ & $3(1-9)$ & $3.5(1.8-10)$ & $0.02^{*}$ \\
\hline Death within 30 days & $6(54.5)$ & $21(21.2)$ & $27(24.5)$ & $0.03^{*}$ \\
\hline
\end{tabular}

ICU: intensive care unit; SOFA: sequential organ failure assessment.

Data are presented as median values (interquartile range) for continuous variables and numbers of cases (\%) for categorical variables.

$P$ values are marked in bold if $<0.05$.

${ }^{a}$ Variables categorised as an ordinal scale in 1-point increments: OR $>1$ is the increase in likelihood of the outcome with a 1-point increase in the factor.

${ }^{\mathrm{b}}$ Days of hospitalisation prior to fungaemia onset.

* $P<0.10$, variable was included in the multivariate analysis of outcomes from non-Candida fungaemia versus candidaemia. 
Table 3 Risk factors predicting non-Candida fungaemia

\begin{tabular}{|c|c|c|c|c|}
\hline \multirow[t]{2}{*}{ Variables } & \multicolumn{2}{|c|}{ Univariate analysis } & \multicolumn{2}{|c|}{ Multivariate analysis } \\
\hline & Crude OR $(95 \% \mathrm{Cl})$ & $P$ value & Adjusted OR $(95 \% \mathrm{Cl})$ & $P$ value \\
\hline \multicolumn{5}{|l|}{ Co-morbidities } \\
\hline Solid malignancy & $0.55(0.46-0.65)$ & $<0.01$ & - & - \\
\hline Chronic kidney disease & $5.40(1.48-19.7)$ & 0.01 & - & - \\
\hline Collagen disease & $3.75(0.84-16.7)$ & 0.09 & $9.00(1.58-51.4)$ & 0.01 \\
\hline \multicolumn{5}{|l|}{ Underlying conditions } \\
\hline Prior bacteraemia & $3.66(1.00-13.4)$ & 0.04 & & \\
\hline Colonisation by causative yeasts & $3.77(0.94-15.1)$ & 0.05 & - & - \\
\hline Renal replacement therapy & $8.33(2.12-32.8)$ & $<0.01$ & $15.0(3.06-73.4)$ & $<0.01$ \\
\hline Corticosteroid therapy & $3.84(1.05-14.1)$ & 0.04 & - & - \\
\hline Chemotherapy & $0.73(0.65-0.82)$ & 0.04 & - & - \\
\hline Other immunosuppressant therapy & $4.27(0.94-19.3)$ & 0.08 & & \\
\hline SOFA score ${ }^{a}$ & $1.13(1.03-1.26)$ & 0.02 & - & - \\
\hline
\end{tabular}

OR: odds ratio; SOFA: sequential organ failure assessment.

$P$ values are marked in bold if $<0.05$.

${ }^{a}$ Variables categorised as an ordinal scale in 1-point increments: OR $>1$ is the increase in likelihood of the outcome with a 1-point increase in the factor.

$P<0.10$, variable was included in the multivariate analysis of outcomes from non-Candida fungaemia versus candidaemia.

micafungin, an echinocandin, is commonly used for initial empirical therapy for yeast fungaemia in our institute.

Among patients with non-Candida fungaemia, one or more co-morbidities were identified in all patients, but none had a solid malignancy. Several factors, including chronic kidney disease, prior bacteraemia, renal replacement therapy, corticosteroid therapy, higher SOFA score, and 30-day mortality, were significantly overrepresented in non-Candida fungaemia. However, independent risk factors associated with non-Candida fungaemia only included collagen disease and renal replacement therapy. Other significant risk factors for non-Candida fungaemia in the univariate analysis might have confounded these associations. Although colonisation by Candida species at multiple sites in the body is commonly recognised as a major risk factor for invasive candidiasis in critically ill patients, Candida colonisation occurs primarily in immunocompromised patients [14]. In this study, colonisation by causative organisms prior to fungaemia onset was noted in $72.7 \%$ of non-Candida fungaemia patients. This finding is intriguing and may be an important clue for predicting the occurrence of non-Candida fungaemia.

Non-Candida fungaemia was significantly associated with 30-day mortality in the univariate analysis but was not an independent predictor in the multivariate analysis. Although this factor could be a confounding variable, the mortality of non-Candida fungaemia was higher than that of candidaemia (54.5\% versus $21.2 \%$ ). This high mortality rate is consistent with previous reports [2,3]. However, the mortality of candidaemia (21.2\%) was lower compared to those of previous reports (50-80\%) [1,7-10]. The difference in the severity of illness might explain this discrepancy. Anunnatsiri et al. reported that patients with non-Candida fungaemia and patients with candidaemia had similar illness severities [2]. In our study, patients with candidaemia had a lower SOFA score than patients with non-Candida fungaemia $(P=0.02)$, although this difference was not significant in the multivariate analysis. Another explanation for this discrepancy is that the clinical intervention by infectious disease physicians contributed to the low mortality of candidaemia in our hospital [15].

Inappropriate therapy is a significant predictor of mortality in fungaemia [7,10,16-19]. Antifungal resistance is common among non-Candida yeasts; thus, treatment options are limited, and the appropriate therapy can be left untried [11]. Initial therapy with antifungals at a high MIC was primarily found in T. asahii fungaemia (75\%). However, the rate of appropriate therapy was similarly low between non-Candida fungaemia and candidaemia when appropriate therapy was defined as treatment with agents at a low MIC within 24 hours (data not shown). These findings suggest that other factors (e.g., background and comorbidities) might contribute to the high mortality of non-Candida fungaemia.

Our results reveal important insights into the epidemiology of non-Candida fungaemia. However, this study had several limitations. The most important limitation was the small sample size of non-Candida fungaemia patients because of low incidence. In addition, other rare opportunistic yeasts (e.g., Rhodotorula species, Saccharomyces species, and Malassezia species) were not detected in this study. Second, due to the retrospective nature of the study, we did not identify the focus or cause of infection in some cases; therefore, we could not address the application of 
appropriate therapy in all non-Candida fungaemia patients. Furthermore, we could not carry out molecular species identification. Finally, this study only evaluated the crude mortality of patients with fungaemia. The development of national databases and well-defined multicentre studies are needed to resolve these limitations.

\section{Conclusions}

In conclusion, although non-Candida fungaemia is a rare cause of yeast fungaemia, it should receive more attention because of its high mortality, particularly among high-risk patients, such as those with collagen disease and those under renal replacement therapy. Prior colonisation by causative organisms may be an important predictor of non-Candida fungaemia.

\section{Competing interests}

The authors declare that they have no competing interests.

\section{Authors' contributions}

MY conceived the study, participated in its design, reviewed the medical records, performed the statistical analysis, and drafted the manuscript. ST and SI participated in the design of the study, coordination, and manuscript preparation. GH, YM, AM, and MN participated in manuscript preparation. All authors read and approved the final manuscript.

\section{Acknowledgements}

This study was presented in part at the Infectious Diseases Society of America $48^{\text {th }}$ annual meeting, Vancouver, Canada, 21-24 October 2010 This work was partly supported by a grant from the Ministry of Health, Labour and Welfare of Japan (H21-Shinkou-Ippan-009 and H23-ShinkouIppan-018). No commercial relationships or potential conflicts of interest exist.

\section{Author details}

'Department of Clinical Laboratory Medicine, Kyoto University Graduate School of Medicine, 54 Shogoin-Kawaharacho, Sakyo-ku, Kyoto 6068507 Japan. ${ }^{2}$ Department of Respiratory Medicine, Kyoto University Graduate School of Medicine, Kyoto, Japan.

Received: 6 September 2012 Accepted: 15 May 2013

Published: 28 May 2013

\section{References}

1. Fridkin SK, Jarvis WR: Epidemiology of nosocomial fungal infections. Clin Microbiol Rev 1996, 9(4):499-511.

2. Anunnatsiri $S$, Chetchotisakd $P$, Mootsikapun P: Fungemia in non-HIVinfected patients: a five-year review. Int J Infect Dis 2009, 13(1):90-96.

3. Chitasombat MN, Kofteridis DP, Jiang Y, Tarrand J, Lewis RE, Kontoyiannis DP: Rare opportunistic (non-Candida, non-Cryptococcus) yeast bloodstream infections in patients with cancer. J Infect 2012, 64(1):68-75.

4. Kontoyiannis DP, Torres HA, Chagua M, Hachem R, Tarrand JJ, Bodey GP, Raad II: Trichosporonosis in a tertiary care cancer center: risk factors, changing spectrum and determinants of outcome. Scand J Infect Dis 2004, 36(8):564-569.

5. Munoz P, Bouza E, Cuenca-Estrella M, Eiros JM, Perez MJ, Sanchez-Somolinos M, Rincon C, Hortal J, Pelaez T: Saccharomyces cerevisiae fungemia: an emerging infectious disease. Clin Infect Dis 2005, 40(11):1625-1634.

6. Riedel DJ, Johnson JK, Forrest GN: Rhodotorula glutinis fungemia in a liverkidney transplant patient. Transpl Infect Dis 2008, 10(3):197-200.

7. Alonso-Valle H, Acha O, Garcia-Palomo JD, Farinas-Alvarez C, FernandezMazarrasa C, Farinas MC: Candidemia in a tertiary care hospital: epidemiology and factors influencing mortality. Eur J Clin Microbiol Infect Dis 2003, 22(4):254-257.

8. Goldani LZ, Mario PS: Candida tropicalis fungemia in a tertiary care hospital. J Infect 2003, 46(3):155-160.
9. Poikonen E, Lyytikainen O, Anttila VJ, Ruutu P: Candidemia in Finland, 1995-1999. Emerg Infect Dis 2003, 9(8):985-990.

10. Viudes A, Peman J, Canton E, Ubeda P, Lopez-Ribot JL, Gobernado M: Candidemia at a tertiary-care hospital: epidemiology, treatment, clinical outcome and risk factors for death. Eur J Clin Microbiol Infect Dis 2002, 21(11):767-774.

11. Miceli MH, Diaz JA, Lee SA: Emerging opportunistic yeast infections. Lancet Infect Dis 2011, 11(2):142-151.

12. National Committee for Clinical Laboratory Standards: Reference method for broth dilution antifungal susceptibility testing of yeasts: approved standard M27-A2. Villanova, PA, USA: NCCLS; 2002.

13. Perfect JR, Dismukes WE, Dromer F, Goldman DL, Graybill JR, Hamill RJ, Harrison TS, Larsen RA, Lortholary O, Nguyen MH, Pappas PG, Powderly WG, Singh N, Sobel JD, Sorrell TC: Clinical practice guidelines for the management of cryptococcal disease: 2010 update by the infectious diseases society of America. Clin Infect Dis 2010, 50(3):291-322.

14. Charles PE, Dalle F, Aube H, Doise JM, Quenot JP, Aho LS, Chavanet P, Blettery B: Candida spp. Colonization significance in critically ill medical patients: a prospective study. Intensive Care Med 2005, 31(3):393-400.

15. Takakura S, Fujihara N, Saito T, Kimoto T, Ito Y, linuma Y, Ichiyama S: Improved clinical outcome of patients with Candida bloodstream infections through direct consultation by infectious diseases physicians in a Japanese university hospital. Infect Control Hosp Epidemiol 2006, 27(9):964-968.

16. Garey KW, Rege M, Pai MP, Mingo DE, Suda KJ, Turpin RS, Bearden DT: Time to initiation of fluconazole therapy impacts mortality in patients with candidemia: a multi-institutional study. Clin Infect Dis 2006, 43(1):25-31.

17. Macphail GL, Taylor GD, Buchanan-Chell M, Ross C, Wilson S, Kureishi A: Epidemiology, treatment and outcome of candidemia: a five-year review at three Canadian hospitals. Mycoses 2002, 45(5-6):141-145.

18. Morrell M, Fraser VJ, Kollef MH: Delaying the empiric treatment of Candida bloodstream infection until positive blood culture results are obtained: a potential risk factor for hospital mortality. Antimicrob Agents Chemother 2005, 49(9):3640-3645.

19. Nolla-Salas J, Sitges-Serra A, Leon-Gil C, Martinez-Gonzalez J, Leon-Regidor MA, Ibanez-Lucia P, Torres-Rodriguez JM: Candidemia in non-neutropenic critically ill patients: analysis of prognostic factors and assessment of systemic antifungal therapy. Study group of fungal infection in the ICU. Intensive Care Med 1997, 23(1):23-30.

doi:10.1186/1471-2334-13-247

Cite this article as: Yamamoto et al.: Clinical characteristics and risk factors of non-Candida fungaemia. BMC Infectious Diseases 2013 13:247.

\section{Submit your next manuscript to BioMed Central and take full advantage of:}

- Convenient online submission

- Thorough peer review

- No space constraints or color figure charges

- Immediate publication on acceptance

- Inclusion in PubMed, CAS, Scopus and Google Scholar

- Research which is freely available for redistribution 